\title{
Talent Training Model of Business English Translation Based on the Graphical Teaching Method
}

\author{
WangMin \\ Nanchang Institute of Technology,Nanchang 330044,China
}

Keywords: Graphical teaching, English translation, HMM corpus, Viterbi decoding, Error tagging.

\begin{abstract}
In order to improve the effect of teaching business English translation and realize the teaching goal of translation talent training, this paper puts forward a pronunciation automatic evaluation method based on phonetic model perception. Combined with expert evaluation method, the method can complete English translation pronunciation error tagging by measuring the similarity between the standard speech and the test speech. Constructing a text corpus of HMM speech automatic evaluation, using Viterbi decoding method designs the phonological similarity judgment method, the text corpus is decomposed into phonetic sequence, and then we can obtain the similarity after the use of training phonetic sequence and test sample data are compared. The teaching experiment is carried out to verify the method, the test results show that the test method finally can output speech tagging errors in the form of graphical results, which provides a visual material for the graphical teaching of English translation.
\end{abstract}

\section{Introduction}

With the development of economic globalization, the talent gap of English translation major is becoming larger and larger, but it is restricted by the learning environment and the teachers' team, there are many deficiencies in the training of English translation professionals [1-3]. The automatic evaluation of the pronunciation is one of the key technologies of business English translation pronunciation aid teaching, the evaluation results can be graphically output, and it can be provided to the teachers in the form of feedback, so as to realize the graphical and visual teaching [4,5]. The modernization evaluation methods are introduced in the process of English translation teaching, we need to consider the needs of practical teaching in the actual measurement, and then we carry out evaluation according to the characteristics of the English translation phonogram, so corpus construction is very important [6-8]. Based on the construction and testing of the corpus, this paper verifies pronunciation automatic evaluation software technology in the feasibility of English translation graphical teaching.

\section{Business English Translation Pronunciation Automatic Evaluation Corpus}

In order to realize the graphical teaching of business English translation, we need to establish the automatic evaluation corpus, the corpus includes three part design, there are training samples, data recording and expert evaluation module, in which the corpus content need to turn in the form of text, the recording reads in accordance with the content, and finally it can be converted into a unified digital audio format [9-11]. Expert marking is mainly to score for the recorded speech, but also to focus tagging on the error, and its basic framework is shown in Figure 1. 


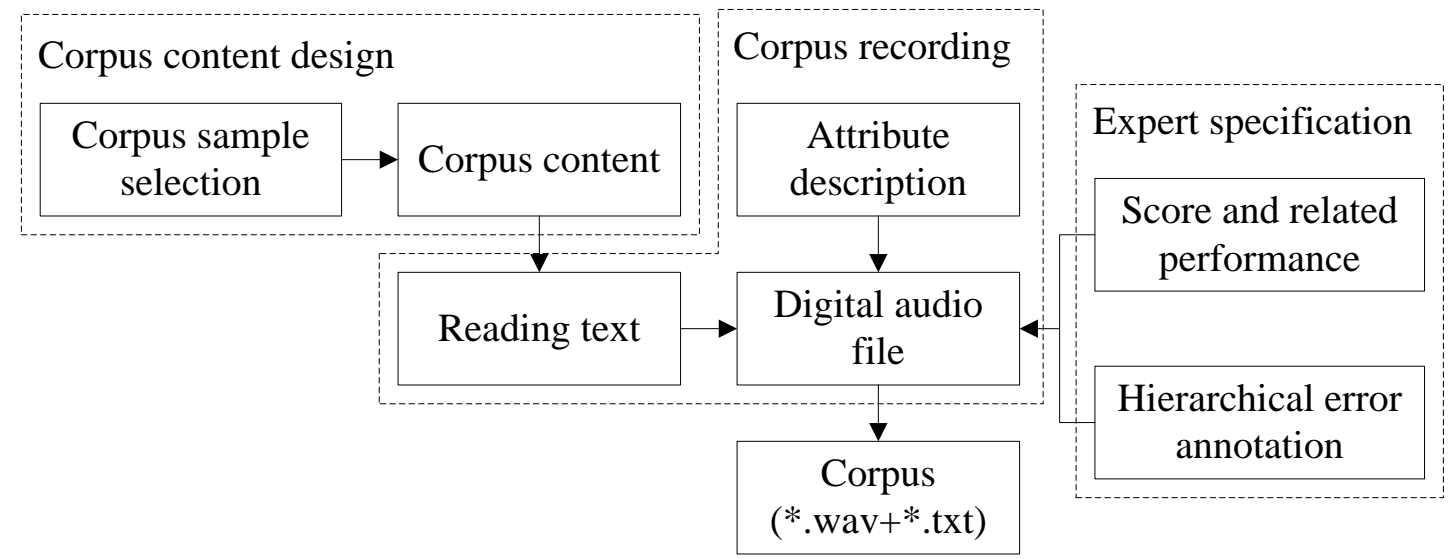

Fig.1 Graphical teaching automatic evaluation corpus framework

In Figure 1, it shows the basic design framework of graphical teaching automatic evaluation corpus, in which corpus is mainly divided into the labeling pronunciation and recording pronunciation, the error evaluation of the pronunciation is divided into automatic evaluation and experts evaluation, and the expert evaluation can improve the accuracy of pronunciation assessment.

\section{English Translation Graphical Teaching Standard Speech Database Modeling}

In order to achieve the graphical teaching mode of business English translation pronunciation practice, it is necessary to establish the standard speech database model [12-14]. This study uses HMM to model the speech, and then speech model training uses the pronunciation data in standard corpus, in which the phonetic model collection can be expressed as

$$
Q_{N p}=\left\{q_{1}, q_{2}, \ldots, q_{N p}\right\}
$$

Wherein $Q_{N p}$ shows the number of all phonetic model in the corpus data. Combined with English translation pronunciation dictionary, pronunciation sequence can be is decomposed into phonetic sequence.

$$
Q^{w}=\left\{q_{w 1}, q_{w 2}, \ldots, q_{w k}\right\}
$$

Wherein $Q^{w}$ shows the number of included phonetic symbols in pronunciation content, $Q^{w} \subseteq Q_{N p}$. The use of $Q^{w}$ can construct phonetic recognition network, the input of the network is sound characteristic data set $O$ for the sample of tested practice, and its expression can be written as

$$
O=\left\{o_{1}, o_{2}, \ldots, o_{T}\right\}
$$

Wherein $T$ shows the frames of the test sample. Using Viterbi decoding can calculate the similarity between the acoustic characteristics of the test samples and the sound characteristics of and training samples. For $\forall q_{w k} \in Q^{w}$, there is

$$
\begin{aligned}
& p\left(O_{q_{w k}} \mid q_{w k}\right)=\max _{\{s, e \subset\{1,2, \ldots, T\}\}}\left(p\left(O_{s}^{e} \mid q_{w k}\right)\right) \\
& \left\{s_{q_{w k}}, e_{w k}\right\}=\max _{\{s, e \subset\{1,2, \ldots, T\}}\left(p\left(O_{s}^{e} \mid q_{w k}\right)\right)
\end{aligned}
$$

Wherein $p\left(O_{q_{w k}} \mid q_{w k}\right)$ shows the similarity degree of measurement sample acoustic features $O_{q_{w k}}$ in the phonetic alphabet $q_{w k}, p\left(O_{s}{ }^{e} \mid q_{w k}\right)$ shows the similarity degree of measurement sample acoustic features $O_{s}{ }^{e}$ in the phonetic alphabet $q_{w k}$, in which they respectively represent the starting and end frame of the test samples. $s_{q_{w k}}$ and $e_{w k}$ respectively represent the start and end frames of the measuring sample acoustic features $O_{q_{w k}}$ in use of $q_{w k}$. 
At last, the pronunciation practice data of automatic evaluation is introduced evaluation module, and then the measured pronunciation will be evaluated [15]. The basis of the test is divided into the expert corpus and the non-expert corpus, and its process is as follows :

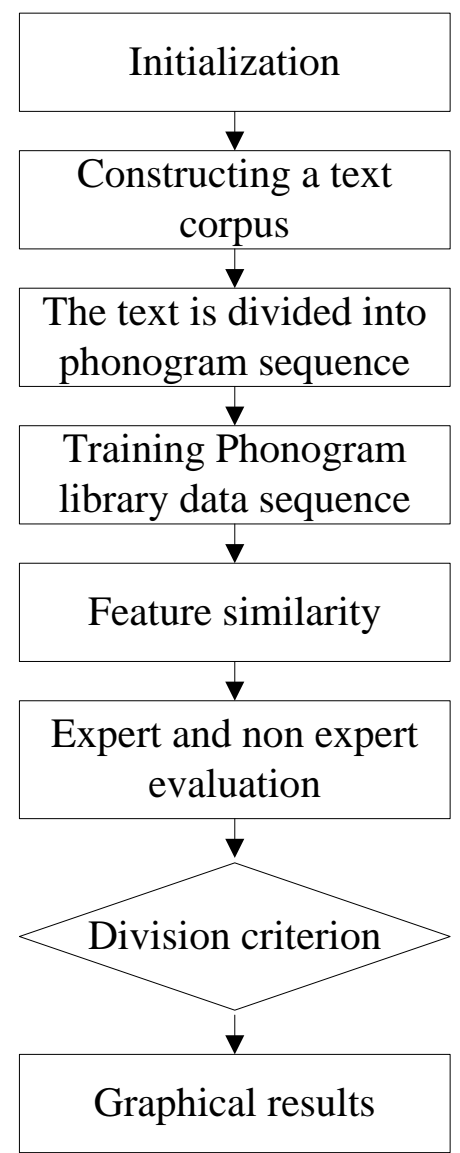

Fig.2 The basic flow chart of translation pronunciation test graphical output

As shown in Figure 2, it shows the basic process of business English translation pronunciation test, the basic steps is to construct a text corpus, and the text corpus is decomposed into a sequence of phonogram. After the training phonetic sequence and test data are compared, we can obtain the similarity, and then to evaluate similarity according to the expert and non-expert system, finally the similarity is output in the form of graphical results. The teachers for business translation courses do the targeted teaching according to the output results, so as to realize the teaching graphic process.

\section{Business Translation English Pronunciation Automatic Evaluation Graphical Teaching}

In order to verify the feasibility of automatic evaluation graphical teaching based on corpus business English pronunciation, this paper carries out experimental teaching for the process . First of all, we record student's pronunciation, and then get corpus information by recording as shown in Table 1.

Table 1. Corpus sample recording information

\begin{tabular}{|c|c|c|c|c|}
\hline Corpus name & Word & Sentence & Sentence group & Total \\
\hline Standard corpus & $6 \mathrm{~h}$ & $8 \mathrm{~h}$ & $12 \mathrm{~h}$ & $26 \mathrm{~h}$ \\
\hline Expert corpus & $6 \mathrm{~h}$ & $8 \mathrm{~h}$ & $12 \mathrm{~h}$ & $26 \mathrm{~h}$ \\
\hline Non expert corpus & $6 \mathrm{~h}$ & $8 \mathrm{~h}$ & $12 \mathrm{~h}$ & $26 \mathrm{~h}$ \\
\hline Test sample & $5.5 \mathrm{~h}$ & $7.6 \mathrm{~h}$ & $11.8 \mathrm{~h}$ & $24.9 \mathrm{~h}$ \\
\hline
\end{tabular}


Table 1 shows the recorded corpus sample information. Automatic evaluation pronunciation data samples use the microphone recording in a quiet classroom, and corpus content is presented the corpus database according to a random sequence. When recording, lips is less than $10 \mathrm{~cm}$ from the standard distance of the microphone, sampling frequency is $16 \mathrm{kHz}$, the precision is 16 bit. After the recording is complete, the data is saved in wav format.

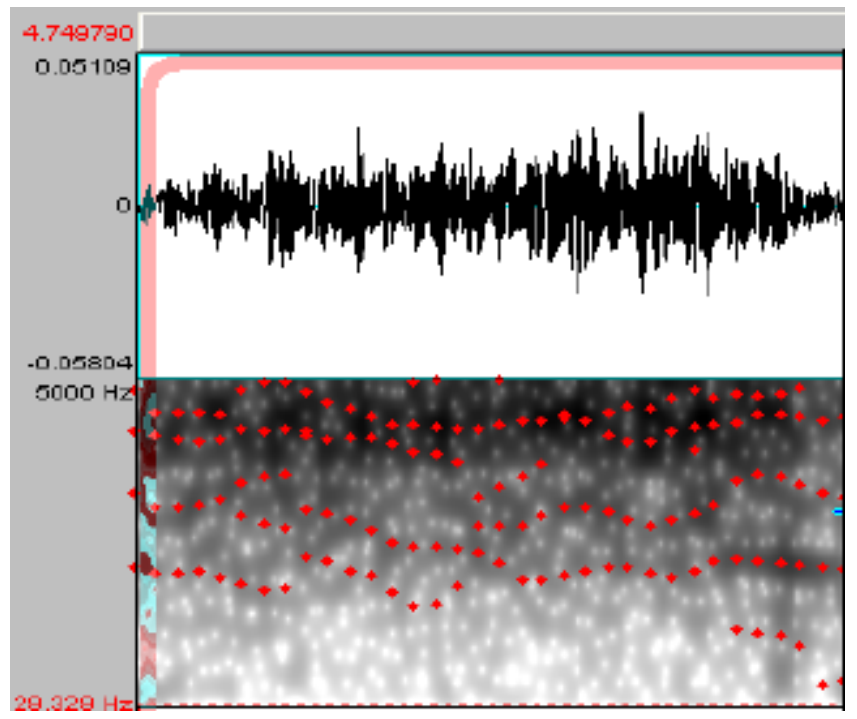

Fig.3Automatic error pronunciation annotation graphical teaching

Figure 3 shows the fragment of business translation English pronunciation automatic testing system, the fragment uses non expert system to carry on pronunciation accuracy test, and the results finally are output by the graphical way. The teacher can combine the figure to carry out targeted correction on the pronunciation, and they can complete the graphical teaching process.

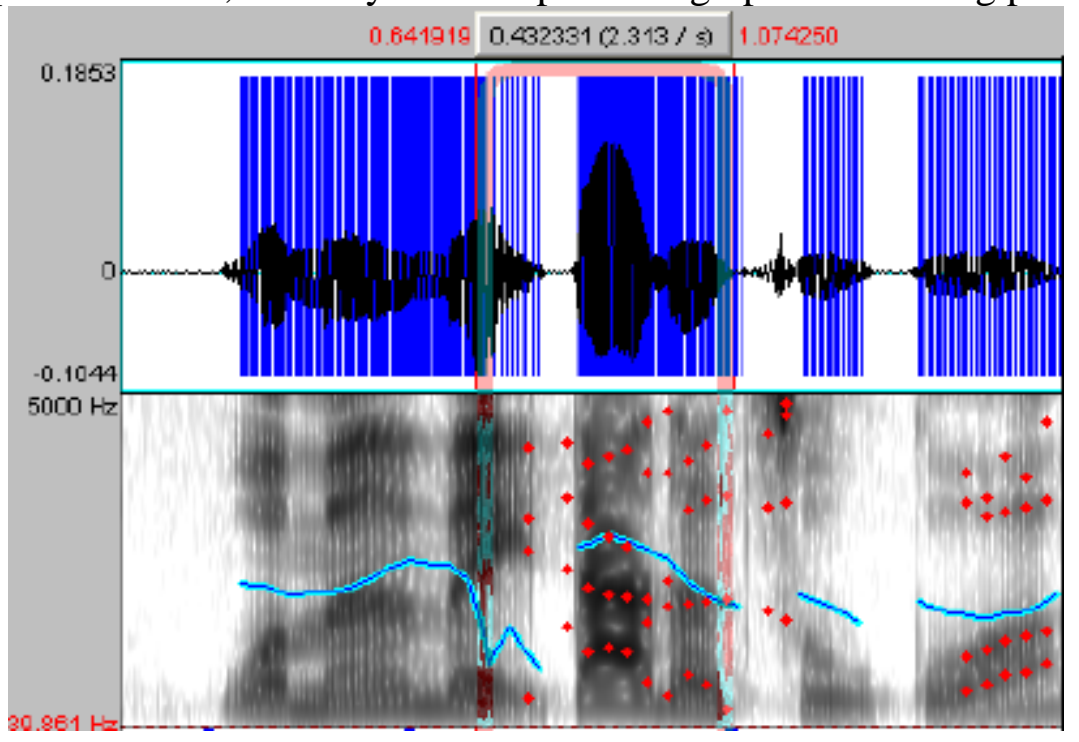

Fig.4 Expert error pronunciation annotation graphical teaching

In order to improve the accuracy and reliability of business English translation pronunciation automatic evaluation corpus annotation, we request five engaged in English translation teaching experts on corpus annotation, in which the error is further determined by the experts, we can obtain expert error pronunciation annotation as shown in Figure 4. Through the further annotation of the error pronunciation, we can improve the basic function of the system and the accuracy of the system, which provides more reliable data for the graphical teaching of business English.

\section{Summary}

According to the situation presents of the of business English translation talent cultivation, this paper presents the automatic pronunciation assessment graphical teaching method based on phonetic 
model perception, the method uses the evaluation software to display visual form for the English translation pronunciation practice error tagging, and gives feedback to teachers in the form of teaching material. The text corpus of HMM pronunciation automatic evaluation is constructed, using the Viterbi decoding method designs the phonological similarity judgment method, finally we verify the feasibility of the method. By the actual teaching experiment, it shows that using this method can mark the sound characteristics of English translation pronunciation errors through automatic evaluation and expert evaluation, which provides reliable graphical teaching data, but also is feasible in the actual teaching.

\section{References}

[1] S.J. Wang, H.Y. Li, D.F Ke, et al. Study on the large scale intelligent evaluation of spoken English for second language learning. Chinese Journal of information science, 2014, 26 (6):142-148.

[2] X.B. Zhou, G.P. Wang. A review of the research on Chinese language as a two language acquisition in the past thirty years. Chinese language learning, 2013(1): 88-95.

[3] D. Deng, Y.J. Wang. Japanese learners on acquisition of "similar vowels" and "unfamiliar vowels" in Chinese mandarin. World Chinese language teaching, 2013(2): 42-48.

[4] J. Xi, W. Jiang, L.J. Zhang et al. Chinese phonological category perception and its development. Chinese Journal of psychology, 2014, 41(7):572-579.

[5] W.Q. Liang, G.L. Wang, J. Liu et al. An evaluation algorithm of pronunciation quality based on phoneme. Journal of Tsinghua University (NATURAL SCIENCE EDITION), 2014, 45(1):5-8.

[6] S. Wei, Q.S. Liu, Y. Hu et al. The electronic system of putonghua proficiency test. Chinese Journal of information science, 2014, 20(6):89-96.

[7] F.Y. Li. The application and thinking of multimedia in English teaching. China information technology education, 2014(8): 43-44.

[8] L.J. Song. Analysis of middle school students' psychological characteristics in English teaching. Educational exploration, 2014(3): 55-56.

[9] J. Song. English teaching should pay attention to the students' emotion. Chapter, 2013(5): 92-93.

[10] Y.M. Zhu. A new thinking on improving students' learning interest and motivation in English teaching. China's off campus education, 2014(4): 105-106.

[11] Y. Yang. The advantages and disadvantages of multimedia teaching in English teaching and learning. Journal of Jilin radio and television university, 2013(6): 146-147.

[12] Y.H. Zhang. A study on the dynamic evaluation model of web writing teaching in college English. Foreign language circles, 2014(4): 73-81.

[13] Y.A. Hu, Z.G. Zheng. Application of wavelet neural network in fault diagnosis of analog circuits. Micro computer information, 2014,22 (16): 206-208

[14] S. Huang, J. Li, H.Y. Wang et al. The pronunciation evaluation algorithm based on the articulation model. Computer application, 2013(S2): 287-289.

[15] Q.S. Liu, S. Wei, Y. Hu et al. The improvement of speech evaluation algorithm based on linguistic knowledge. Chinese Journal of information science, 2013, 21(4):92-96. 